Vol. 14, No. 1: $31-42$

\title{
GPU-Based Neighbor-Search Algorithm for Particle Simulations
}

\author{
Serkan Bayraktar, Uğur Güdükbay, and Bülent Özgüç \\ Department of Computer Engineering, Bilkent University
}

\begin{abstract}
This paper presents a neighbor-search technique to be used in a GPU-based particle simulation framework. Neighbor searching is usually the most computationally expensive step in particle simulations. The usual approach is to subdivide the simulation space to speed up neighbor search. Here, we present a grid-based neighbor-search technique designed to work on programmable graphics hardware.
\end{abstract}

\section{Introduction}

Particle systems are a widely used simulation technique in computer graphics [Miller and Pearce 89, Reeves 83, Terzopoulos et al. 91, Tonnesen 91]. Many natural phenomena, such as fabric, fluid, fire, cloud, smoke, etc., have been modeled and simulated by using particle systems. In most of these cases, particles interact with each other only if they are closer than a predetermined distance. Thus, determining the neighbor particles speeds up the whole simulation.

Locating particle neighbors dominates the run-time of a particle-based simulation system. Space subdivision methods have been developed to achieve faster proximity detection. These methods usually employ a uniform grid [Kipfer and Westermann 06, Teschner et al. 03] discretizing the simulation space to improve the performance of contact detection. Bounding volumes [Teschner et al. 04], and binary space partitioning (BSP) trees [Melax 00] are other popular methods of improving contact detection performance. 
In this work, we present a grid-based neighbor-search technique for programmable graphics hardware (GPU). We use this method to implement smoothed particle hydrodynamics (SPH) [Gingold and Monaghan 77, Monaghan 92] entirely on the GPU. Our neighbor-search technique is suitable to be used in conjunction with any other particle-based simulation system.

\section{Fluid Simulation}

Our implementation uses the SPH paradigm to simulate fluid behavior. The $\mathrm{SPH}$ method has been used in astrophysics and computational physics to simulate natural phenomena, such as smoke, fluid, and debris. It is a particlebased method and has been used in computer graphics to simulate lava flow, free fluid flow, hair behavior, and bubble and froth generation [Cleary et al. 07, Hadap and Magnenat-Thalmann 01, Müller et al. 03, Müller et al. 04].

In the SPH method, fluid is represented by a set of particles that carry various fluid properties such as mass, velocity, and density. These properties are distributed around the particle according to an interpolation function (kernel function) whose finite support is $h$ (kernel radius). For each point $x$ in simulation space, the value of a fluid property can be computed by interpolating the contributions of fluid particles residing within a spherical region with radius $h$ and centered at $x$. For more detailed information on simulating fluid using SPH, the reader may refer to [Müller et al. 03].

Typically, the non-penetration boundary condition in SPH applications is enforced by inserting stationary boundary particles to construct a layer of width $h$, which exerts repulsive forces on the fluid particles [Monaghan 92].

\section{Neighbor Search}

Space subdivision is one of the ways to speed up the SPH force computation. Search for potential inter-particle contacts is done within the grid cells and between immediate neighbors, thus improving the whole simulation speed. We employ the same basic principle in this work to achieve a fast and reliable neighbor search.

\subsection{CPU-Based Neighbor Search}

The first step of the algorithm is to compute a one-dimensional grid coordinate of each particle. This is done by discretizing particle coordinates with respect to a virtual grid of cell size $h$ and obtaining integral positions $\left(i_{x}, i_{y}, i_{z}\right)$. These 


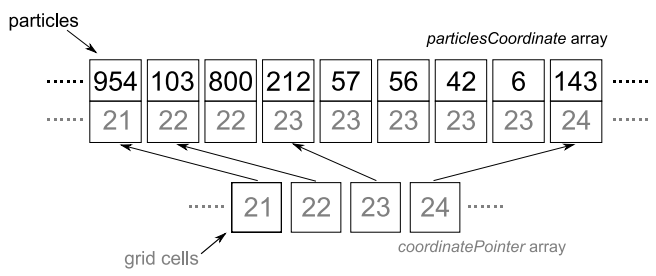

Figure 1. The sorted particleCoordinate array and coordinatePointer array.

integral coordinates are converted to $1 \mathrm{D}$ coordinates by Equation 1:

$$
i_{x}+i_{y} \times \text { grid_width }+i_{z} \times \text { grid_width } \times \text { grid_height } .
$$

After sorting the particles with respect to their grid coordinates using a radix sort, we determine the number of particles each cell hosts. Figure 1 illustrates the corresponding data structure. The particleCoordinate array stores the sorted particles and the coordinatePointer array has pointers to the array particle Coordinate for fast access.

The potential neighbors of a particle are determined by following the pointers from coordinatePointer and by scanning the array particleCoordinate. Constant offsets are employed to access each of the 26 neighboring cells of the particle's host cell. For example, the $(i-1)$ th cell is the left neighbor of $i$ th cell and the $(i+$ grid_width $)$ th cell is the upper neighbor of the $i$ th cell. Figure 2 illustrates the algorithm where potential neighbors of particle 57 are being searched (within a virtual grid 30 cells wide). The algorithm scans the particles of cell 23 (the host cell of particle 57), cell 24 (the right neighbor cell), and cell 53 (the upper-neighboring cell). To consider each potential neighbor pair exactly once, we scan particles in descending order and terminate when we reach a particle with a smaller ID.

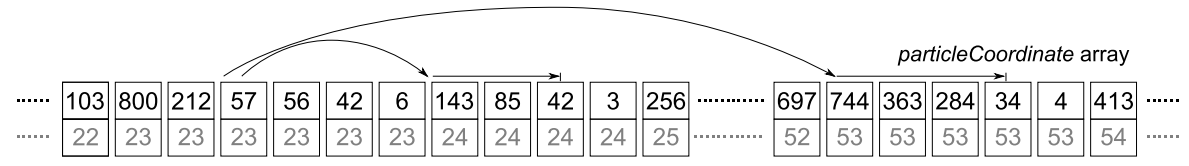

Figure 2. Potential neighbors of particle 57 are being searched.

\subsection{GPU-Based Neighbor Search}

Particle-based simulations, including SPH, are perfect candidates to be implemented on GPUs because of the GPUs ability to process multiple particles in parallel [Pharr and Fernando 05]. The biggest challenge of implementing a particle-based simulation on a GPU is to detect particle proximity efficiently. 
This is because of the fact that the fragment shaders that we use as the main processing unit are not capable of scatter. That is, they cannot write a value to a memory location for a computed address since fragment programs run using precomputed texture addresses only, and these addresses cannot be changed by the fragment program itself. This limitation makes several basic algorithmic operations (such as counting, sorting, finding maximum and minimum) difficult.

One of the common methods to overcome this limitation is to use a uniform grid to subdivide the simulation space. Kipfer et al. [Kipfer et al. 04] use a uniform grid and sorting mechanism to detect inter-particle collisions on the GPU. Purcell et al. [Purcell et al. 03] also use a sorting-based grid method. They employ a stencil buffer for dealing with multiple photons residing in the same cell. Harada et al. [Harada et al. 07] present a SPH-based fluid simulation system. Their system uses bucket textures to represent a 3D grid structure and make an efficient neighbor search. One limitation of their system is that it can only handle up to four particles within a grid cell.

\section{Implementing the Proposed Method on the GPU}

Figure 3 illustrates the work flow of the GPU-based particle simulation that employs the presented technique to compute the particle neighborhood information. Each white rectangle in the figure represents a rendering pass by a fragment program and each gray rectangle represents an RGBA float texture.

\subsection{Grid-Map Generation}

Algorithm 1 outlines the grid-map generation process. The first two steps of the algorithm compute 1D grid coordinates of particles (Equation (1)), store this information in the grid-coordinate texture (texGridCoor) along with particle IDs, and sort the texture with respect to grid coordinates. The sorting phase employs the bitonic sort [Purcell et al. 03], which makes $\log ^{2} N$ passes over the texture, where $N$ is the number of particles.

The next step computes the grid-map texture (texGridMap), which corresponds to the coordinatePointer array in Section 3.1. The fragment program makes a binary search in the sorted grid-coordinate texture for each grid cell to determine the first particle residing in the grid cell and stores this information in the $\mathrm{R}$ channel of the grid-map texture.

The final step counts the total number of particles belonging to each grid cell and stores this information in the $\mathrm{G}$ channel. Then, a fragment program takes these values in the grid-map texture as input, checks all 26 neighbors 


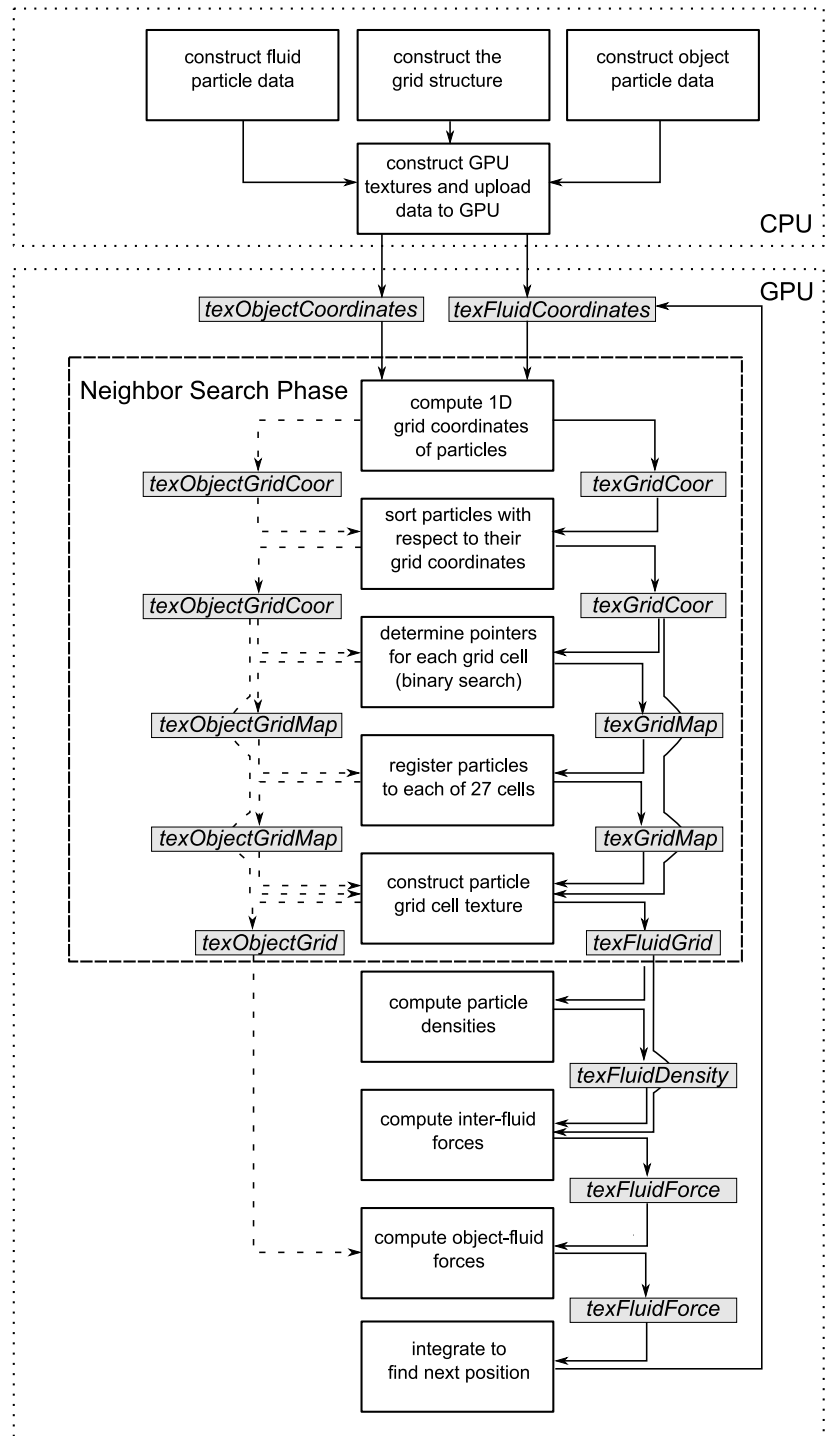

Figure 3. The framework for the GPU-based SPH implementation.

of each grid cell, and determines the total number of particles belonging to each grid cell and its immediate neighbors. This information is stored in the B channel of the grid-map texture. Finally, there is one more pass on the grid-map texture to accumulate the values in the B channel and store this sum in the alpha channel. 


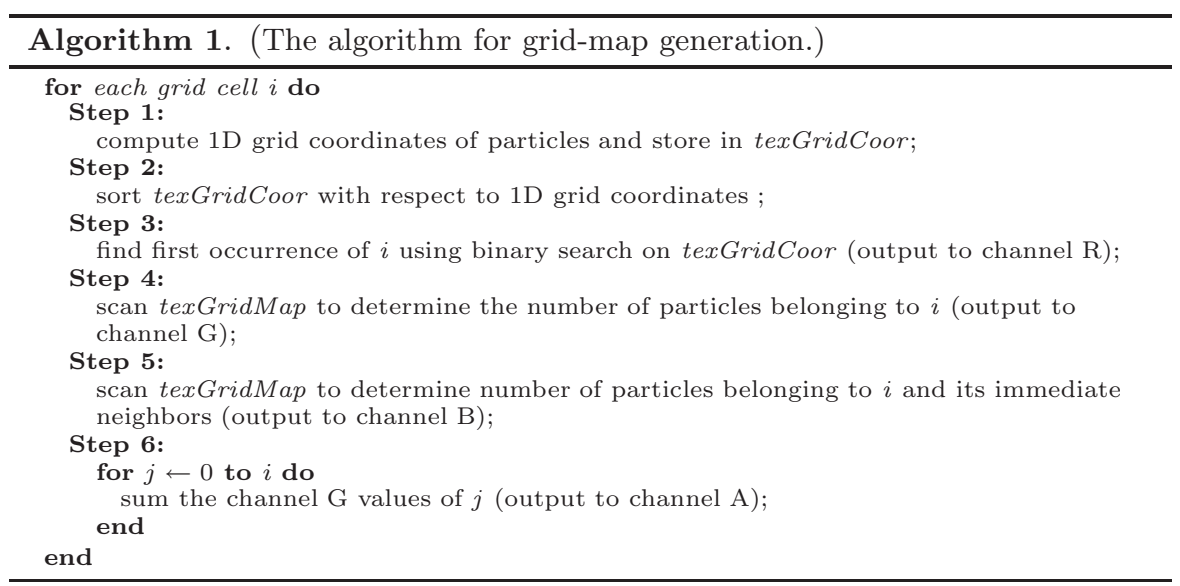

Figure 4 depicts a sample grid-map texture. In this figure,

- the values in the $\mathrm{R}$ channel point to the first occurrence of the grid cell within the grid-coordinate texture texGridCoor;

- the values in the $\mathrm{G}$ channel are the total number of the particles hosted within the grid cell;

- the $\mathrm{B}$ values are the total number of particles hosted in the grid cell itself and its immediate neighbors;

- the alpha-channel values point to the first occurrence of the grid cell with the fluid-grid texture (texFluidGrid), which is generated afterwards.

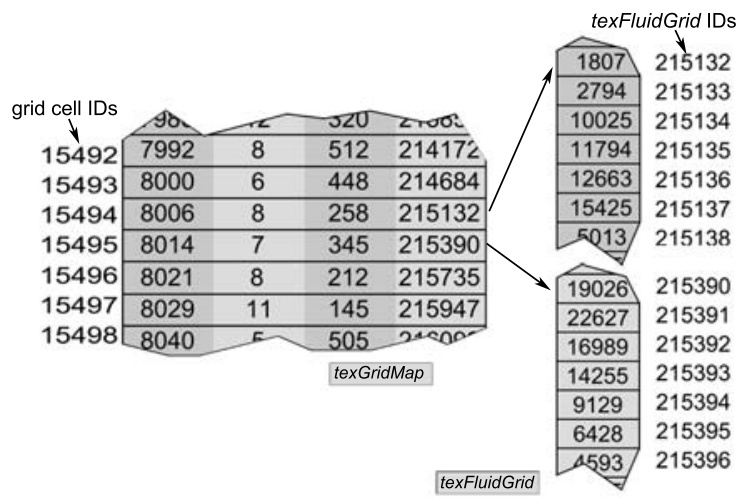

Figure 4. Grid-map texture and fluid-grid texture. 


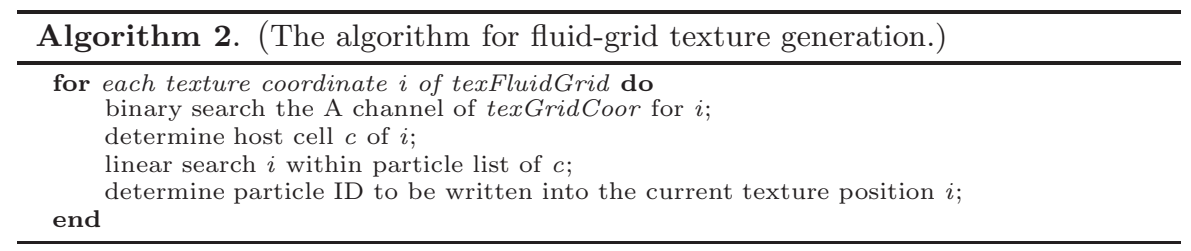

\subsection{Generating Fluid-Grid Texture and Neighbor Lookup}

Algorithm 2 outlines the fluid-grid texture generation. Fluid-grid texture (texFluidGrid) is similar to grid-coordinate texture (texGridCoor) in the sense that it contains particle IDs sorted with respect to their $1 \mathrm{D}$ grid coordinates. The difference between texGridCoor and texFluidGrid is that the latter has an entry for the particle host cell and, additionally, an entry for each of the immediate neighbor cells of the host cell. Each particle appears up to 27 times in texFluidGrid; once for the host cell and 26 times (which could be less if the host cell lies on the grid boundary) for each neighboring cell.

To construct texFluidGrid, the fragment program has to decide which particle ID is to be stored in the current texture coordinate. Basically, the fragment program determines the grid cell hosting the particle by searching the particles using the alpha channel of the grid-map texture texGridMap. Then, by following the pointers of the grid cell to the grid-coordinate texture texGridCoor, the fragment program finds the appropriate particle ID. For example, assume that the current texture coordinate is 215,425 and the current texGridMap is as shown in Figure 4. Binary searching for this coordinate in the alpha channel of the texGridMap reveals that the particle resides in the neighborhood of the grid cell 15,495. Then, to determine the particle ID to store in the current location, we go through the list of hosted particles in the neighborhood of grid cell 15,495.

After generating the textures texGridMap and texFluidGrid, finding possible neighbors of a particle is a simple task. We use the particle's 1D grid ID to look up the pointer to texFluidGrid. For example, if the 1D grid cell coordinate of particle $i$ is 15,494 , then $i$ has 258 potential neighbors starting with 1,807 (see Figure 4).

\subsection{Rigid Body-Fluid Particle Neighbor Search}

The dashed arrows in Figure 3 show that the rigid-body particles (and solid boundaries) go through the same process to generate particle-grid information. In our case, since the rigid objects do not move during the simulation, the object textures are generated once at the outset. If we choose to move the boundaries or to add moving objects represented by a set of points, then we have to generate particle-grid information for each particle at every time step. 


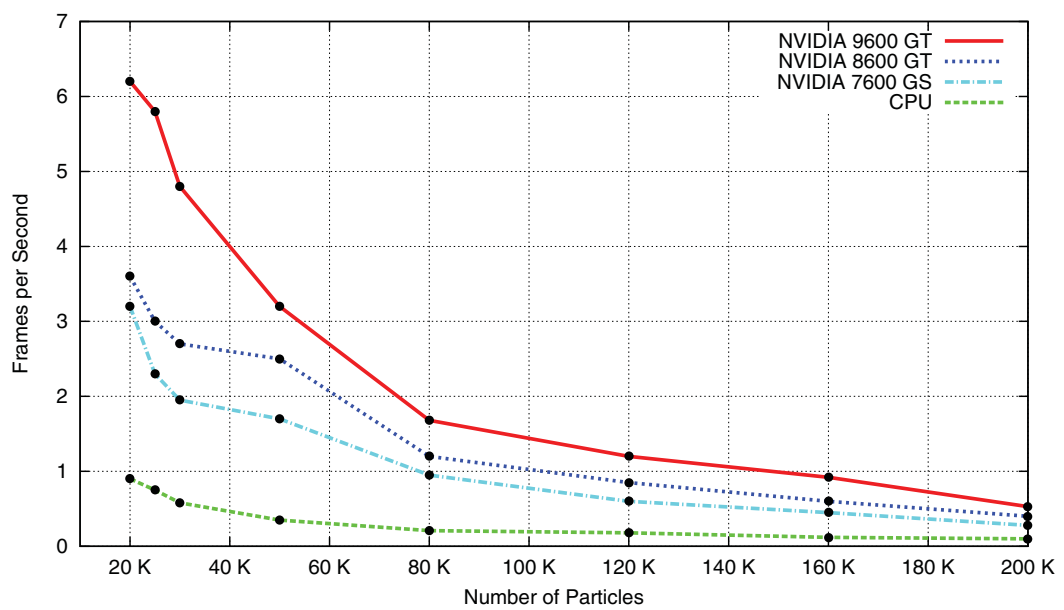

Figure 5. The frame rates of the breaking-dam simulation for different number of particles.

\section{Examples}

Figure 5 depicts the frame rates of the breaking-dam simulation (Figure 6) on three different NVIDIA ${ }^{\mathrm{TM}}$ graphics boards and CPU. The test platform is a $\mathrm{PC}$ equipped with an Athlon ${ }^{\mathrm{TM}} 64 \mathrm{X} 2$ Dual Core processor and $3 \mathrm{~GB}$ RAM. The specifications of the tested GPUs are given in Table 1. One of the key observations is that the memory bandwidth is not a crucial factor for the performance since the texture data is uploaded to the GPU memory once, and there are virtually no memory transfers between the system memory and the GPU memory during the simulation. On the other hand, the graphics clock speed and the number of microprocessors have a major impact on the performance because of the parallel nature of the algorithm. Because the CPU version uses Radix Sort that has linear asymptotic complexity, one would expect that the CPU version behaves better for very large datasets. For all of the practical datasets we use for experimentation (up to 200K), the GPU version performs better.

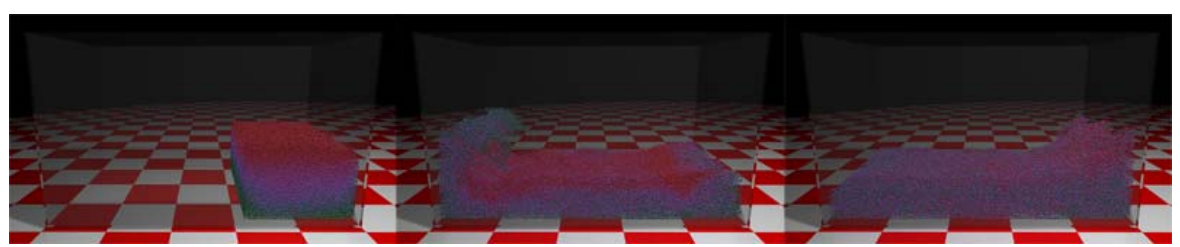

Figure 6. Still frames from the breaking-dam simulation (150K fluid particles). 


\begin{tabular}{|l|c|c|c|}
\cline { 2 - 4 } \multicolumn{1}{c|}{} & \multicolumn{3}{c|}{ GPU } \\
\hline & $\mathbf{7 9 0 0}$ GS & $\mathbf{8 6 0 0}$ GT & $\mathbf{9 6 0 0}$ GT \\
\hline Processor cores & 20 & 32 & 64 \\
\hline Graphics clock (MHz) & 450 & 540 & 650 \\
\hline Processor clock (MHz) & 1320 & 1190 & 1625 \\
\hline Memory clock (MHz) & 700 & 700 & 900 \\
\hline Memory configuration (GDDR3) & $256 \mathrm{MB}$ & $1024 \mathrm{MB}$ & $1024 \mathrm{MB}$ \\
\hline Memory interface width & 256 -bit & 128 -bit & 256 -bit \\
\hline Memory bandwidth (GB/sec) & 42.2 & 22.4 & 57.6 \\
\hline
\end{tabular}

Table 1. The specifications of the GPUs used in the dam-breaking scene.

\begin{tabular}{|l|c|c|}
\hline Texture & $\begin{array}{c}\text { Texture Type } \\
\text { (channels) }\end{array}$ & $\begin{array}{c}\text { Memory } \\
\text { used }\end{array}$ \\
\hline Particle coordinate & RGB & $24 \mathrm{MB}$ \\
\hline Particle velocity & RGB & $24 \mathrm{MB}$ \\
\hline Particle acceleration & RGB & $24 \mathrm{MB}$ \\
\hline Particle density & $\mathrm{R}$ & $8 \mathrm{MB}$ \\
\hline Particle-grid coordinate (texGridCoor $)$ & $\mathrm{RG}$ & $16 \mathrm{MB}$ \\
\hline Grid-map texture (texGridMap) & RGBA & $32 \mathrm{MB}$ \\
\hline Fluid-grid texture (texFluidGrid) & $\mathrm{RG}$ & $432 \mathrm{MB}$ \\
\hline
\end{tabular}

Table 2. The memory requirements of a SPH simulation based on the proposed neighbor-search algorithm. The texture dimension is $2048 \times 1024$.

The memory requirements of the dam-breaking simulation are shown in Table 2. The size of textures allocated to store particle information is $2048 \times$ 1024 (storage space for more than two million particles). The total memory requirement of the simulation is $560 \mathrm{MB}$.

Figure 7 shows still frames from a simulation where fluid particles flow down onto a fountain model. The fluid body consists of $64 \mathrm{~K}$ particles at the end of the simulation, and the fountain object consists of about $64 \mathrm{~K}$ particles. The frame rate we obtain for the simulation is approximately 2 fps on an NVIDIA

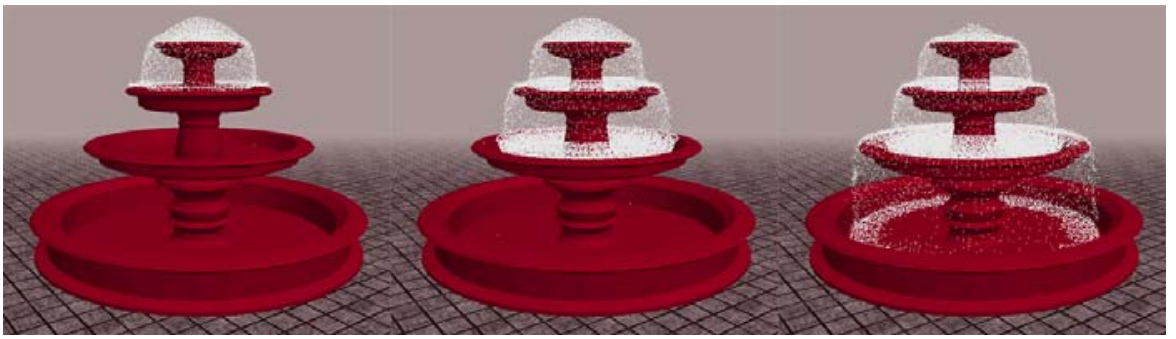

Figure 7. Still frames from the fountain simulation. 


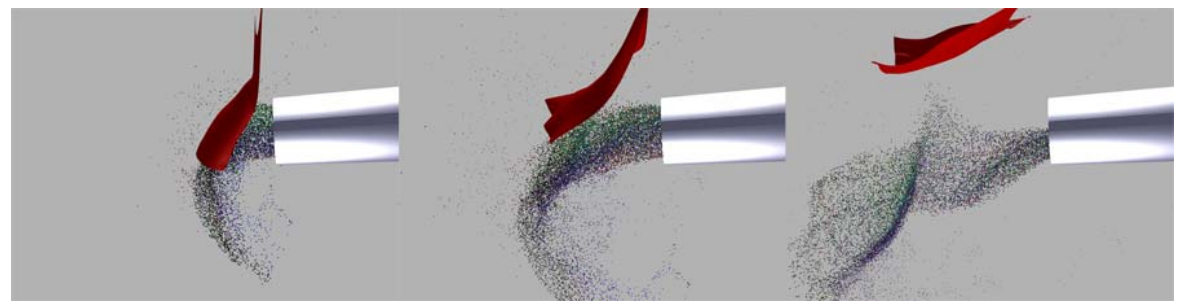

Figure 8. Fluid particles flow into a cloth mesh.

$8600 \mathrm{GT}$. If we use the neighbor-search algorithm for the CPU, we obtain a frame rate of approximately 0.5 fps.

In Figure 8, fluid particles flow into a cloth mesh that is simulated by a massspring network. The cloth mesh is composed of $20 \mathrm{~K}$ particles, and collisions between the cloth and the fluid particles are detected by the proposed method. The frame rate is 5 fps on an NVIDIA 8600 GT.

\section{Conclusion}

This paper presents a neighbor-search algorithm to be used in particle-based simulations. The algorithm sorts particles with respect to their grid coordinates and registers them to their neighboring grid cells. It then looks for potential particle proximities by using this grid-particle map. The main focus of the paper is to propose a method to overcome the current inability of fragment shaders to do scatter operations.

The primary strength of the proposed method is that it does not, unlike the existing techniques, make an assumption about the particle density, the radius of the neighbor search, the number of grid cells, and the grid-cell size. This is especially important when simulating compressible fluids and dense natural phenomena, e.g., sand, smoke, and soil. The algorithm yields optimum performance if the radius of the neighbor search is one cell.

The main shortcoming of the method is that it is slower than the similar sorting-based grid methods for fixed grid particle density, e.g., [Harada et al. 07]. This is because of the expensive grid-map generation phase in our method. As a future work, the method can be implemented by using Compute Unified Device Architecture (CUDA), which has scatter capabilities.

\section{References}

[Cleary et al. 07] P. W. Cleary, S. H. Pyo, M. Prakash, and B. K. Koo. "Bubbling and Frothing Liquids." ACM Transactions on Graphics 26:3 (2007), Article No. 97,6 pages. 
[Gingold and Monaghan 77] R. A. Gingold and J. J. Monaghan. "Smoothed Particle Hydrodynamics: Theory and Application to Non-Spherical Stars." Monthly Notices of the Royal Astronomical Society 181 (1977), 375-389.

[Hadap and Magnenat-Thalmann 01] S. Hadap and N. Magnenat-Thalmann. "Modeling Dynamic Hair as a Continuum." Computer Graphics Forum 20:3 (2001), 329-338.

[Harada et al. 07] T. Harada, S. Koshizuka, and Y. Kawaguchi. "Smoothed Particle Hydrodynamics on GPUs." In Proc. of Computer Graphics International, pp. 63-70. Geneva: Computer Graphics Society, 2007.

[Kipfer and Westermann 06] P. Kipfer and R. Westermann. "Realistic and Interactive Simulation of Rivers." In GI '06: Proceedings of Graphics Interface '06, pp. 41-48. Toronto: Canadian Information Processing Society, 2006.

[Kipfer et al. 04] P. Kipfer, M. Segal, and R. Westermann. "UberFlow: A GPUBased Particle Engine." In Proc. of the ACM SIGGRAPH/EUROGRAPHICS Conference on Graphics Hardware, pp. 115-122. Aire-la-Ville, Switzerland: Eurographics Association, 2004.

[Melax 00] S. Melax. "Dynamic Plane Shifting BSP Traversal." In Proc. of Graphics Interface '00, pp. 213-220. Toronto: Canadian Information Processing Society, 2000 .

[Miller and Pearce 89] G. S. P. Miller and A. Pearce. "Globular Dynamics: A Connected Particle System for Animating Viscous Fluids." Computers and Graphics 13:3 (1989), 305-309.

[Monaghan 92] J. J. Monaghan. "Smoothed Particle Hydrodynamics." Annual Review of Astronomy and Astrophysics 30 (1992), 543-574.

[Müller et al. 03] M. Müller, D. Charypar, and M. Gross. "Particle-Based Fluid Simulation for Interactive Applications." In Proc. of the ACM SIGGRAPH/Eurographics Symposium on Computer Animation, pp. 154-159. Airela-Ville, Switzerland: Eurographics Association, 2003.

[Müller et al. 04] M. Müller, S. Schirm, M. Teschner, B. Heidelberger, and M. Gross. "Interaction of Fluids with Deformable Solids." Journal Computer Animation and Virtual Worlds 15:3-4 (2004), 159-171.

[Pharr and Fernando 05] M. Pharr and R. Fernando. GPU Gems 2: Programming Techniques for High-Performance Graphics and General-Purpose Computation. Reading, MA: Addison-Wesley Professional, 2005.

[Purcell et al. 03] T. J. Purcell, C. Donner, M. Cammarano, H. W. Jensen, and P. Hanrahan. "Photon Mapping on Programmable Graphics Hardware." In Proc. of the ACM SIGGRAPH/EUROGRAPHICS Conference on Graphics Hardware, pp. 41-50. Aire-la-Ville, Switzerland: Eurographics Association, 2003.

[Reeves 83] W. T. Reeves. "Particle Systems - A Technique for Modeling a Class of Fuzzy Objects." ACM Transactions on Graphics 2:2 (1983), 91-108. 
[Terzopoulos et al. 91] D. Terzopoulos, J. Platt, and K. Fleischer. "Heating and Melting Deformable Models." The Journal of Visualization and Computer Animation 2:2 (1991), 68-73.

[Teschner et al. 03] M. Teschner, B. Heidelberger, M. Müller, D. Pomerantes, and M. H. Gross. "Optimized Spatial Hashing for Collision Detection of Deformable Objects." In Proc. of Vision, Modeling, Visualization (VMV'03), pp. 47-54. Aka GmbH: Heidelberg, Germany, 2003.

[Teschner et al. 04] M. Teschner, S. Kimmerle, G. Zachmann, B. Heidelberger, L. Raghupathi, A. Fuhrmann, M.-P. Cani, F. Faure, N. Magnetat-Thalmann, and W. Strasser. "Collision Detection for Deformable Objects." In Eurographics State-of-the-Art Report (EG-STAR), pp. 119-139. Aire-la-Ville, Switzerland: Eurographics Association, 2004.

[Tonnesen 91] D. Tonnesen. "Modeling Liquids and Solids Using Thermal Particles." In Proc. of Graphics Interface '91, pp. 255-262. Toronto: Canadian Information Processing Society, 1991.

\section{Web Information:}

Additional material can be found online at http://jgt.akpeters.com/papers/ BayraktarEtAl09/

Serkan Bayraktar, Department of Computer Engineering, Bilkent University, 06800, Bilkent, Ankara, Turkey (serkan@cs.bilkent.edu.tr)

Uğur Güdükbay, Department of Computer Engineering, Bilkent University, 06800, Bilkent, Ankara, Turkey (gudukbay@cs.bilkent.edu.tr)

Bülent Özgüç, Department of Computer Engineering, Bilkent University, 06800, Bilkent, Ankara, Turkey (ozguc@bilkent.edu.tr)

Received June 27, 2008; accepted in revised form April 17, 2009. 